\title{
Supervised Integrated Screening of Low-Back Pain Patients by a Neurologist
} A Randomized Clinical Trial

\author{
Ludwien Meeuwesen, Ph.D., Frits J. Huyse, Gerrit T. Koopmans, and \\ Jan J. Heimans
}

\begin{abstract}
In a randomized, controlled design, the effect of psychiatric consultation intervention in medical outpatients with low-back pain ( $\mathrm{N}=104$ ) was assessed for the patients' subjective well-being and satisfaction of general practitioners (GPs) in their cooperation with the neurologists. The goal of the intervention was an integrated approach towards the patients' symptoms and the improvement of the cooperation between primary and secondary medical health services. The intervention was designed at the health care provider level; the psychiatrist did not see or examine the patient. The 104 patients were subdivided into an intervention group $(\mathrm{N}=50)$ and a control group $(\mathrm{N}=54)$. The major outcome measures were the psychological status of the patient and degree of the GPs' satisfaction concerning the cooperation with the medical specialists. After 6 months follow-up, there was a greater overall decline of symptoms in the patient intervention group compared with the control group. However, it was not possible to specify this effect. Contrary to the hypotheses, GPs in the control group were as satisfied about the cooperation with the neurologist as their colleagues in the intervention group. Yet, the information in the specialists' letters of the intervention group was more often in agreement with the integrated model carried out. It is concluded that great diversity of the target group of patients
\end{abstract}

This project was funded by the Research Center Primary/ Secondary Health Care, Free University Hospital, Amsterdam, The Netherlands. The mission of the Center focuses on the development of models to enhance the collaboration between the primary and secondary health care system.

A parallel study was conducted in abdominal pain patients in an internal outpatient clinic $[33,38]$.

Research Center Primary/Secondary Health Care (L.M.), Consultation Liaison Psychiatry Service (F.J.H.), and Department of Neurology (J.J.H.), the Free University Hospital, Amsterdam, The Netherlands, and Department of Health Care Policy and Management, Erasmus University of Rotterdam, The Netherlands (G.T.K.)

Address reprint requests to: $\mathrm{L}$. Meeuwesen, Ph.D., Utrecht University, Department of General Social Sciences, P.O. Box 80.140, 3508 TC Utrecht, The Netherlands. and the focus on the neurologists' behavior may explain the lack of hypothesized effects. It is recommended that the target group be more specifically defined, and that a more intensioe intervention might prove to be more effectioe. (c) 1996 Elsevier Science Inc.

\section{Introduction}

Primary care physicians and somatic specialists are frequently consulted by patients with low back pain (LBP). In a general practice in the Netherlands, LBP (without radiation) has an annual incidence of about $3.5 \%$ and an annual prevalence of approximately $4 \%$; for LBP with radiation these figures are $0.5 \%$ and $1 \%$, respectively [1]. Of these incidence rates, the complaints become chronic in $18 \%$ of the cases and a majority of the patients were not able to participate in their prior job [2].

LBP is not only a problem because of its consequences, but also because of its complicated etiology. In addition to functional reasons, there are four groups of physical diagnoses for LBP. These concern syndromes 1) of muscles and joints without radicular strain symptoms, 2) of muscles and joints with radicular strain symptoms, 3) affecting the hip, or 4) referred abdominal pain. A precise pathoanatomical diagnosis can be given in only $10 \%-20 \%$ of patients with acute LBP; in most cases diagnoses are unspecific (e.g., lumbosacral strain) [3]. Although the causes of LBP are diverse, it is commonly found that low back pain, especially in chronic cases, is associated with psychological distress, alcohol abuse related disonders, depressive symptoms, and failure to cope $[4,5]$. These comorbidities are associated with a more frequent use of health services [6], such as more medical proce- 
dures, higher costs and increased hospital stay [7, $8]$, and response to medical treatment $[9,10]$. Psychiatric consultation is a potentially cost-effective method for improving mental health treatment and reducing unnecessary use of health care in dealing with patients with combined physical and psychiatric complaints [11-13]).

In addition, factors in the health care system itself contribute to processes of somatization, i.e., a lack of cooperation and a functional division of tasks among health care workers [14]. In the Netherlands, there is a two-tiered approach to patient screening and treatment [15]. The first line is the domain of the general practitioners (GPs), each of whom cover approximately 2500 patients. The secondary medical specialist services are only consulted by GPs for more specific diagnosis and/or treatment skills. When a patient is referred by primary to secondary care services, the communication between these two lines of health care is generally poor $[14,16]$. It is in the interest of all parties to improve the cooperation between the two services, especially in the management of somatization [17-21]. The emphasis on communication between primary and secondary care services and the consequent integrated approach to the symptoms provide paradigms for the management of patients with somatic and psychological symptoms such as LBP $[18,22]$.

The aims of this study are to apply the principles of direct communication at health care provider level in a randomized controlled design in patients with LBP seen by neurologists in the secondary care service, and to assess the effects on patients as well as physicians. The following hypotheses with regard to the new approach will be tested: 1) the subjective well-being of the patients in the experimental group in terms of an improvement in psychological symptoms, or at least stable psychological symptoms, compared with the control group; 2) GPs and neurologists are more satisfied with the new approach.

\section{Method}

\section{Subjects and Procedure}

The setting of this study was the medical outpatient clinic at the Free University Hospital, Amsterdam, which serves about 400 GPs. New patients referred with LBP were asked to take part in the study. Exclusion criteria were age limits below 18 or above 65 years, illiteracy or poor proficiency in the Dutch language, and clear evidence of somatic pathology such as cancer or referred hip pain or abdominal pathology. The target complaints were low-back pain with or without irradiation pain in one (or both) $\operatorname{leg}(\mathrm{s}) . '$

Randomization of the patients occurred at the medical specialist level. During the experiment, a team of four neurologists was allocated to the experimental group, and another team of four neurologists to the control group. For this experiment, both teams provided care independently of each other in order to minimize carry-over effects. Of the 93 GPs involved, 86 had just one patient represented in the sample. Of the 119 patients who were eligible for the study, 104 agreed to participate (50 in the intervention group and 54 in the control group). Reasons for dropouts were 1) eight patients refused to cooperate, 2) two patients were dismissed by their neurologist immediately after the first or second visit, and 3) five patients were missing for logistic or administrative reasons. Consequently, the dropout rate was $13 \%$. Figure 1 shows a flowchart of the distribution of subjects. Comparison of the eligible group and the group in the study revealed no differences in sociodemographic factors.

\section{Baseline Assessment}

Patients were selected during their first visit to the clinic by a doctor not participating in this study. The patients were then contacted by letter, which explained the aim of the study, asking them to participate. During their second visit to the clinic, those who consented were assessed as follows: 1) a routine neurological screening for LBP including medical history, physical assessment, laboratory and, if considered necessary, ancillary procedures; 2) a structured biomedical and brief psychosocial history, including the medical history over the past 10 years; 3 ) an assessment of the number of health care providers involved; and 4) patients reported the following set of baseline measures concerning psychological symptoms:

- 58-item somatization scale derived from physical complaints observed in DSM-III-R somatoform disorders [23]

- Functional Status subscale of the Quality-of-Life Assessment [24], a self-report measure of functional impairment $(\mathrm{FBI})$, i.e., of the degree to which one's present health status interferes with normal daily activities such as walking, climbing 


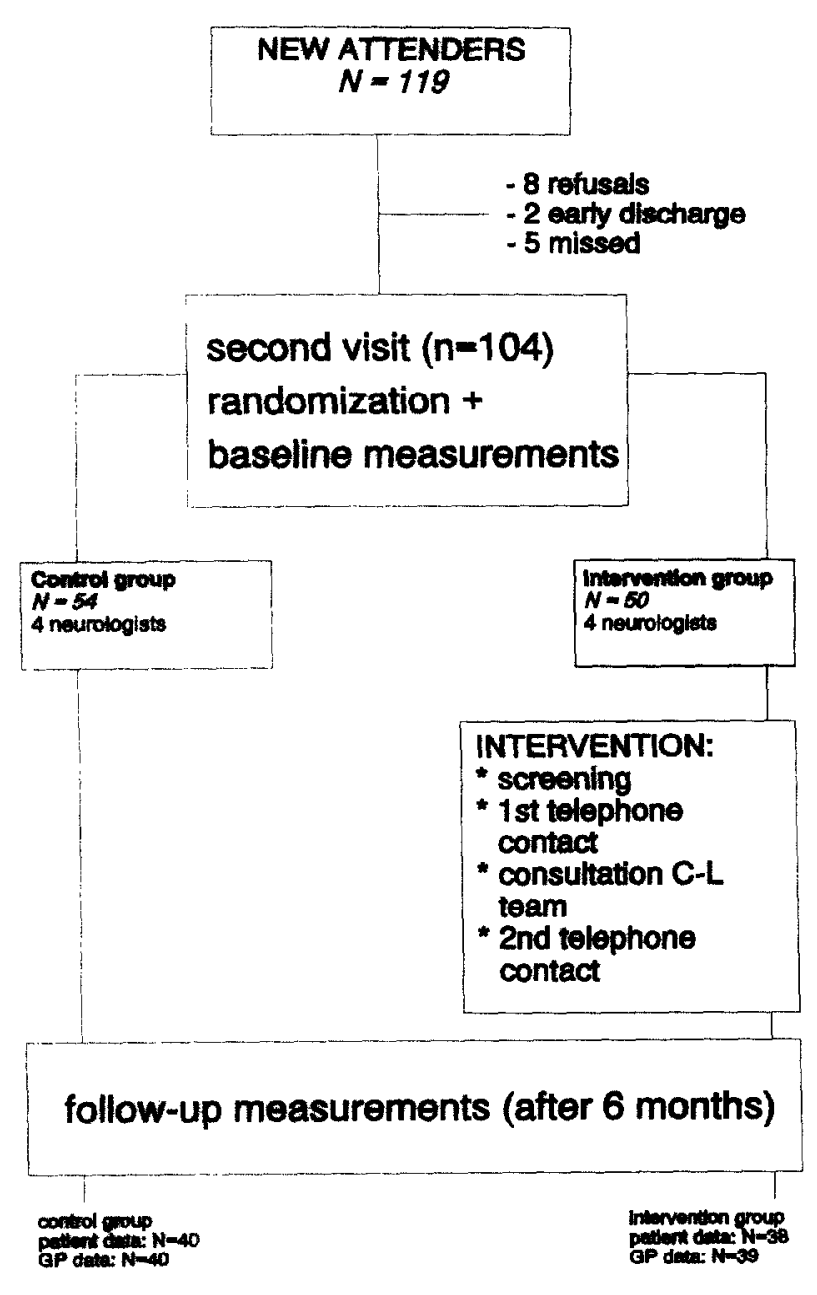

Figure 1. Study design.

stairs, eating, shopping, performance of household activities, and so forth

- 28-Item version of the General Health Questionnaire or $G H Q-28[25,26]$ as a self-report instrument of psychological components of ill-health

- Four dimensions of the revised Dutch version of the Symptom Checklist-90 [27], a self-report inventory reflecting patients' psychological symptom patterns, anxiety, depression, somatization, and psychoneuroticism.

Patients were divided into chronic and nonchronic patients. The criterion for chronicity was set at 6 months $[4,10]$.

\section{Intervention}

During a 12-month period, an intervention focusing on integrated treatment and cooperation through close communication between first and second health care line was initiated in a randomized, experimental design, including all newly admitted patients who met the criteria.

The intervention used was largely based on Pincus' model for patterns of cooperation between primary care providers and psychiatrists, with a focus on clear management and organizational agreement on the content of care and its related tasks and communication between the providers of care [18]. The protocol for this experiment was designed by neurologists, general practitioners, and a psychiatrist. In the experiment, the psychiatrist was a supervisor; he did not see or examine the patient. His recommendations were based entirely on the information provided by the neurologist and the baseline measures collected by a test psychologist. An essential element in the intervention was a structured communication by telephone, initiated by the neurologist (see Figure 1). First, there was telephone communication of 5-10 minutes between the medical specialist and GP, focusing on the verification of the biopsychosocial data provided by the patient, and checking the reason for referral. The next step was a weekly consultation session in a team, consisting of neurologists, a psychiatrist, and a test psychologist; the findings from the physical assessment, the additional psychosocial assessment including the scales and those obtained through the telephone conversations with the GP were reviewed. Subsequently, the team decided whether or not physical and/or psychological factors played an important role in the current state, and whether or not the current health care service delivery was adequate. As a result, a treatment plan selected from a menu of operationalized interventions was recommended [28]. The main possibilities were 1) referral back to the GP (autonomous care by GP), 2) joint care (GP and neurologist), 3) autonomous care by neurologist or psychiatrist, or 4) referral to a mental health care institution. This plan was communicated by the neurologist to the GP in a second telephone communication, resulting in a confirmed or somewhat adjusted care plan for the patient. This was communicated to the patient by the ncurologist.

\section{Follow-up Data}

Intervention and control group patients were interviewed 6 months after randomization. The assessment contained the psychometric questionnaires assessing psychological symptoms, as described above. The patients' response rates for the follow- 
up questionnaires were $76 \%$ (experimental) and $74 \%$ (control), nearly the same for both groups.

The extent of the GP satisfaction with the new intervention was assessed by GPs rating three questions with a five-point scale. The three items referred to the quality of the 'cooperation,' 'communication' and 'patient treatment.' The response rates were $78 \%$ (experimental) and $74 \%$ (control).

All the medical specialists in the intervention group completed a similar questionnaire about the new treatment and cooperation. To assess the impact of the implementation of the integrated model on the specialists' behavior, a content analysis of the specialists' letters was performed $[29,30]$. The analysis focused on the conclusion section and treatment recommendations of the letters. For example, the types of information in the conclusion included somatic information (biological reason for the complaint), psychological information (psychosocial reason for the complaint), and information referring to case management principles in a complex organization of health care (e.g., multiple providers).

The protocol requested a considerable change in procedures. Consequently, the protocol adherence by the neurologist was assessed: the first telephone interview took place in $88 \%$ and the second in $82 \%$ of cases. The second telephone communication may be considered essential because of this interview arrangements were made with the GP regarding the treatment plan. Consequently, a differentiation of the intervention in levels of implementation will provide some insight into how process variables might influence the effectiveness of the intervention. Three levels of implementation of the experimental protocol were distinguished: 1) no implementation: no second telephone call; 2) semiimplementation: the second telephone call took place but was not recalled by the GP; and 3) full implementation: the second telephone took place and was recalled by the GP.

\section{Data Analyses}

Before analyzing intervention effects on patients, checks for homogeneity of variance were performed. Some analyses resulted in a heterogeneous variance effect. Despite this violation of homogeneity of variance, all variables were included in the subsequent analyses because as long as the group sizes are approximately equal (largest/smallest $<1.5) F$ is robust against heterogeneous variances [31]. To trace a possible differential effect of the in- tervention and control condition on the dependent variables, a two-way (condition * time) multivariate variance analysis (MANOVA) was performed.

To trace a possible differential effect on GP satisfaction, one-sided $t$-tests were performed. For comparisons of the content of the specialist discharge letters, $\chi^{2}$ tests were performed.

\section{Results}

Table 1 shows the sociodemographics of the outpatient sample. The mean (SD) age of the patient group was $41.7 \pm 11.8$ years. There were more women than men in the cohort. In order to check for selection bias, homogeneity tests were done and showed that the two groups were similar on all the sociodemographic variables examined.

Table 2 shows the psychological well-being.

At baseline, the mean scores of the groups on the measures used were mostly the same for both groups, except for the significantly higher mean scores on the SCL depression and psychoneuroticism in the control group compared with the experimental group (two-sided $t$-tests, $p<0.05$ ). According to Lobo et al. [32], recommending a GHQ cutoff score of $6 / 7$ for a comparable group of patients, $46 \%$ of the intervention patients and $54 \%$ of the control group were at high risk for a psychiatric disorder. Furthermore, $19 \%$ of the total patient group had a number of psychoneurotic complaints, which was above the average, high, or very high with a corrected norm. This percentage was somewhat higher in the control than in the intervention group ( $24 \%$ and $14 \%$, respectively). No significant correlations were found between degree of psychological and physical well-being and (non)chronicity of LBP.

\section{Intervention Effects: Patients}

There was a significant two-way multivariate interaction $(F[7,57]=2.90 ; p=0.01)$ : there was a greater overall decline in psychological symptoms in the patient intervention group. Multivariate simple main effects showed a general and similar decline in both conditions (experimental: $F[7,57]=173.28 ; p$ $<0.001$; control: $\mathrm{F}[7,57]=171.52 ; p<0.001)$. There was a time main effect: $\mathrm{F}[7,55]=341.97 ; p<0.001$. Subsequently, separate two-way univariate analyses of variance per scale were performed (see Table 3 ). The measures did not show significant two-way interactions; however, there was a simple main effect in FBI symptoms (Functional Impairment 
Table 1. Sociodemographic profile of the medical outpatients sample with low-back pain

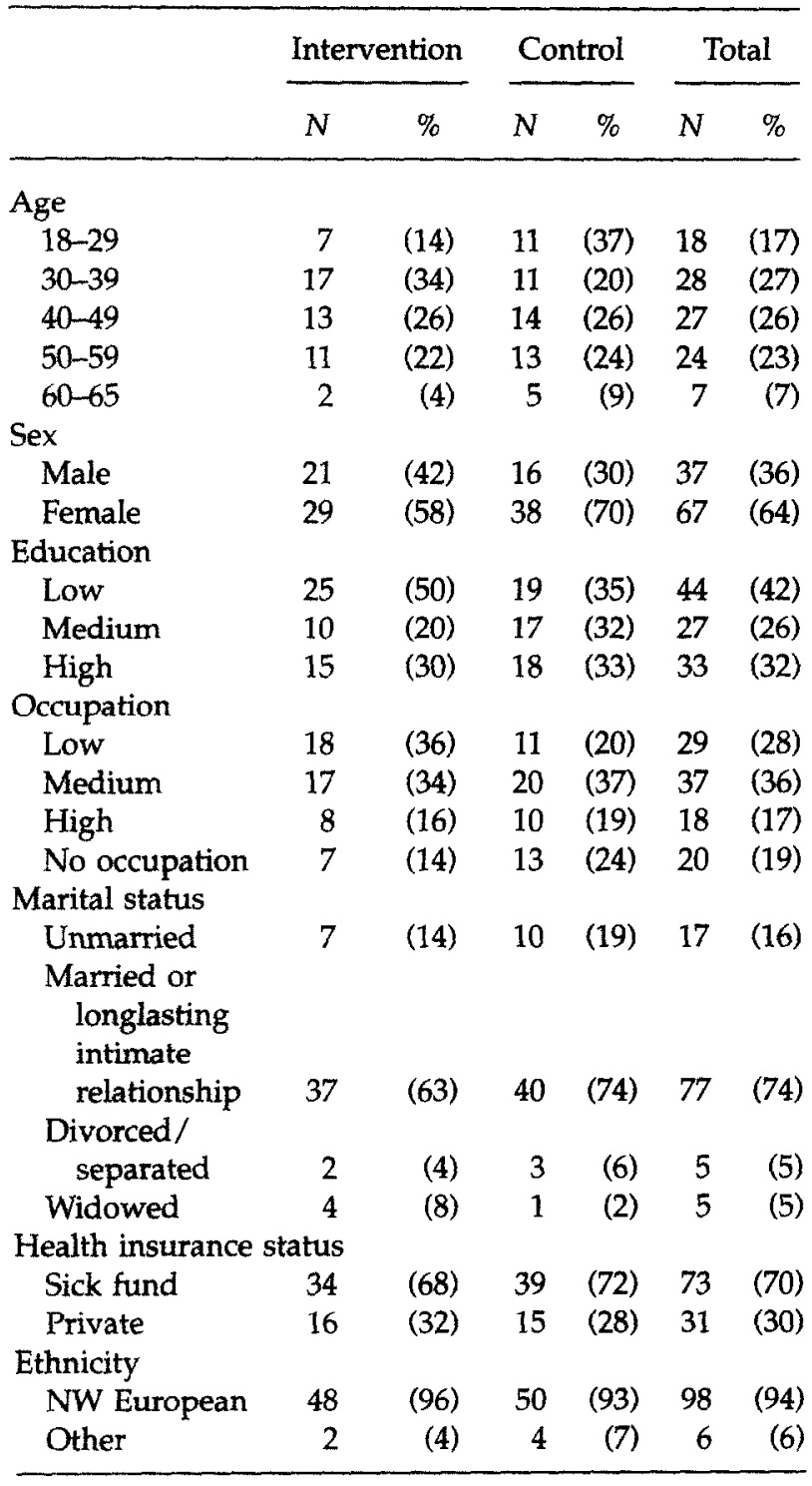

Scale) in both groups and a tendential significant decline in GHQ symptoms in the intervention group. Controlling for the effects of chronicity by means of analysis of covariance did not change the results. Also, additional analysis of covariance with the factor "risk of a psychiatric disorder" (GHQ-28) as a covariate did not influence the results.

In conclusion, although there is a greater overall decline in psychological symptoms in the patient intervention group over time, this result is difficult to interpret. It is not clear to which specific scale(s) this interaction effect can be contributed. Both groups show an improvement in two measures in particular: the functional impairment (FBI) and the general feeling of health (GHQ) (tendential). This improvement was somewhat higher in the experimental condition. There was no effect on the other measures.

\section{Satisfaction of General Practitioners}

All GPs (dropouts excluded) recognized the importance of the intervention model and appreciated being involved in the care planning process as soon as possible after referral. Contrary to the hypothesis, the GPs $(N=40)$ of the patients in the intervention group were as satisfied with the treatment plan $(X=$ 3.4 and $X=3.7)$, the cooperation ( $X=2.9$ and $X=$ $2.9)$, and communication with the neurologists $(X=$ 3.0 and $X=2.8)$ as their colleagues in the control group $(N=39)$ (one-sided $t$-tests). Whereas the scale values range from 1 (=least satisfied) to 5 ( $=$ most satisfied), the mean scores are rather neutral

\section{Opinion and Behavior of Neurologists}

All neurologists $(N=4)$ in the experimental group underscored the importance of the protocol and the cooperation with the GPs, and regarded the psychiatric supervision as positive. In practice, however, when implementing the model, they were restricted by reasons of time and experience, which affected their satisfaction with the model. No effect on the collaboration with the GPs was reported, being concordant with the GPs' reports mentioned above.

The specialists' discharge letters for the experimental group showed a significant difference in the type of information provided in the paragraph describing the conclusion (Figure 2). This concerned information on psychopathology ( $21 \%$ vs $0 \%$, intervention and control group, respectively). With regard to the recommendations on subsequent case management, there were no differences between the two groups.

\section{Impact of the Protocol Implementation}

According to the classification described above (see Method), the protocol had not been implemented in 5 patients, only half in 17 , and fully in 14 (in another 14 cases insufficient data were available). Reasons for noncompliance were diverse: lack of time, work pressure, difficulty in reaching the GP by telephone, feelings of insecurity in handling the patients' emotions, lack of knowledge about specific patterns of psychopathology. The relatively low protocol ad- 
Table 2. Baseline characteristics of intervention, control, and total group of low-back pain patients: health and mental health status

\begin{tabular}{|c|c|c|c|c|c|c|c|c|c|c|c|}
\hline & \multicolumn{3}{|c|}{ Intervention } & \multicolumn{3}{|c|}{ Control } & \multicolumn{5}{|c|}{ Total group } \\
\hline & Mean & (SD) & $N$ & Mean & $(\mathrm{SD})$ & $N$ & Mean & $(\mathrm{SD})$ & Minimum & Maximum & $N$ \\
\hline SCL-SOM & 20.5 & $(4.2)$ & 50 & 22.3 & $(7.0)$ & 52 & 21.40 & 5.86 & 12.00 & 45.00 & 102 \\
\hline DSM-SOM & 7.4 & (4.3) & 50 & 9.4 & (6.8) & 53 & 8.43 & 5.75 & 1.00 & 36.00 & 103 \\
\hline FBI & 5.9 & (2.4) & 48 & 5.4 & (2.9) & 54 & 5.63 & 2.68 & 0.00 & 11.00 & 102 \\
\hline ANG & 13.0 & (3.4) & 50 & 13.7 & $(4.5)$ & 52 & 13.36 & 3.99 & 10.00 & 32.00 & 102 \\
\hline DEP & 20.9 & (5.5) & 50 & 25.4 & $(10.3)$ & $52^{*}$ & 23.14 & 8.56 & 16.00 & 56.00 & 101 \\
\hline PSNEUR & 120.8 & (17.9) & 50 & 134.5 & $(37.1)$ & $51^{*}$ & 127.71 & 29.87 & 91.00 & 262.00 & 101 \\
\hline GHQ-28 & 6.9 & $(4.8)$ & 48 & 8.6 & (6.5) & 54 & 7.78 & 5.77 & 0.00 & 27.00 & 102 \\
\hline
\end{tabular}

${ }^{*} P<0.05$ (two-sided $t$-test).

SCL-SOM = SCL-90 somatization subscale; DSM-SOM = physical complaints derived from DSM-III-R somatoform disorders; FBI = functional impairment scale; ANG = SCL-90 anxiety subscale; DEP = SCL-90 depression subscale; PSNEUR = SCL-90 overall-score psychoneuroticism; GHQ-28 = General Health Questionnaire.

herence by the neurologists (39\%) might reflect the complex multiphase character of the intervention.

Tracing a possible differential effect of the level of implementation on the dependent variables, there were no correlations between the baseline subjective well-being [except for functional impairment $(r$ $=0.27, p=0.05)]$, the satisfaction of the GP, the content of the specialist letters, or the chronicity factor. In other words, neurologists are not inclined to implement the protocol more fully in the case of patients with greater psychological pathology. In addition, a differential effect of the implementation level on the dependent variables in terms of symptom decline could not be demonstrated (one-way tests).

\section{Discussion}

First, a substantial proportion of these patients appears to have a probable psychiatric disorder $(50 \%)$, as assessed by GHQ. This is consistent with earlier findings $[4,26,33]$. Second, close liaison between pri-

Table 3. Univariate treatment effects on psychological symptoms of patients with low back pain

\begin{tabular}{lllrr}
\hline Measures & $\begin{array}{c}\text { Two-way interaction } \\
\text { (treatment }\end{array}$ & \multicolumn{1}{c}{$\begin{array}{c}\text { Simple main effects } \\
\text { over time }\end{array}$} & M1 & M2 \\
\hline SCL-SOM & $\mathrm{F}[1,69]<1$ & $\mathrm{I}: \mathrm{F}[1,69]<1$ & 20.7 & 19.9 \\
DSM-SOM & $\mathrm{F}[1,69]<1$ & $\mathrm{C}: \mathrm{F}[1,69]<1$ & 20.9 & 21.4 \\
& & $\mathrm{I}: \mathrm{F}[1,69]<1$ & 7.7 & 7.2 \\
FBI & $\mathrm{F}[1,70]$ & $\mathrm{C}: \mathrm{F}[1,69]<1$ & 9.2 & 9.5 \\
& & $\mathrm{I}: \mathrm{F}[1,70]=12.87 ; p=0.001$ & 5.9 & 4.3 \\
ANG & $\mathrm{F}[1,70]<1$ & $\mathrm{C}: \mathrm{F}[1,70]=4.33 ; p=0.041$ & 5.3 & 4.4 \\
& & $\mathrm{I}: \mathrm{F}[1,70]<1$ & 12.8 & 12.9 \\
DEP & $\mathrm{F}[1,69]<1$ & $\mathrm{C}: \mathrm{F}[1,70]=1.09 ; \mathrm{n} . \mathrm{s}$. & 13.3 & 13.8 \\
& & $\mathrm{I}: \mathrm{F}[1,69]<1$ & 20.3 & 20.4 \\
PSNEUR & $\mathrm{F}[1,69]<1$ & $\mathrm{C}: \mathrm{F}[1,69]<1$ & 25.0 & 24.8 \\
& & $\mathrm{I}: \mathrm{F}[1,69]<1$ & 119.8 & 119.7 \\
GHQ-28 & $\mathrm{F}[1,72]<1$ & $\mathrm{C}: \mathrm{F}[1,69]<1$ & 131.5 & 132.6 \\
& & $\mathrm{I}: \mathrm{F}[1,72]=3.31 ; p=0.07$ & 6.1 & 4.1 \\
& & $\mathrm{C}: \mathrm{F}[1,72]=2.48 ; p=0.12$ & 9.3 & 7.6 \\
\hline
\end{tabular}

Abbreviations the same as in Table 2.

M1 = mean scores at baseline; $M 2$ = mean scores at follow-up ( 6 months later); $I$ = intervention group; $\mathrm{C}=$ control group. 


\section{Content discharge letters: neurologitat $(n-86)$.}

Information

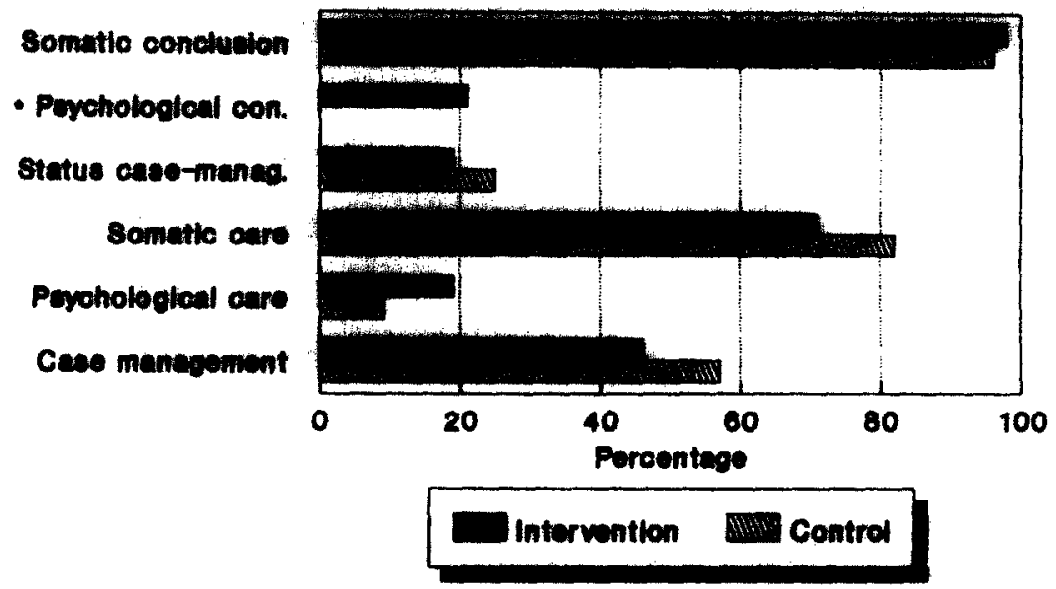

Figure 2. Content discharge letters: neurologist $(n=85)$. The first three categories refer to information in the conclusion segment of the specialist discharge letters ('somatic', 'psychological' and 'case management', respectively). The last three categories refer to treatment recommendations given in the specialist discharge letters ('somatic', 'psychological' and 'case management', respectively). mary and secondary care services resulted in a significant greater overall decline in psychological symptoms in the patient intervention group. However, this effect did not hold true for any of the separate dependent variables. Third, another study of the same sample of patients reported that a reduction in medical consumption could not be demonstrated [37]. Fourth, close liaison did not result in greater satisfaction among the GPs in terms of communication and cooperation with the specialist. Fifth, close liaison was associated with improvement in the content of specialists' discharge letters, i.e., significantly more information was included about the psychological aspects of illness and follow-up arrangements.

Katon and Gonzales [20] recently reviewed the methodology of C-L intervention studies. In the context of this review, the present research can be regarded as a second generation study, yet without formal psychiatric screening. According to this classification, second generation studies include screening followed by structured psychiatric interviews and feedback through written protocols and treatment recommendations. Katon and Gonzales discuss intervention characteristics that can reduce the estimated effect sizes in these studies.

The following three aspects of the intervention used in this study must be considered in understanding the findings [34]. First, the level or focus of the intervention: Is the intervention focused on the provider, being the neurologist, the psychiatrist, and the GP, or on the patient? This study was funded by the Research Center Primary/Secondary Health Care of the Free University of Amsterdam. This center has a primary focus on the development of models to enhance the flexibility of the interaction between the primary and secondary health care system. Consequently, the main focus of the study has been on the change of the behavior of the providers. Therefore, any effect of the intervention on the patient was mediated through the providers. As a result, their adherence to the intervention protocol was crucial. There were two levels of protocol adherence: 1) the adherence by the neurologist to the communication protocol, and 2) the concordance of the GP with the recommendations provided by the neurologist. The first was controlled in the study, the second was not. However, a serious flaw in this study is that the full intervention protocol was carried out in only 14 patients (39\%) in the treatment group. Complete intervention is essential to determine the impact of an experiment. Distinguishing three implementation groups, as we did in this study, is one way to shed some light on this issue. The adherence problem is even more complex as a result of the interdependency of the behavior of several providers, who had to adhere to their part of the protocol. For somatic specialists, psychiatric comorbidity may have a rather low priority, even if they are sympathetic towards the intervention. This and possible nonconcordance of the GP might have resulted in a reduced effect size, a type II error.

Second, the intensity of the intervention, meaning 
does the intervention, being consultation or cotreatment, influence the effect size [35]? As argued, the primary focus of the intervention was the provider, therefore, this study is an example of a consultation study. The main goal of the intervention was to enhance the control of the GP on the treatment of the patient, thereby securing his pivotal role in the treatment of the patient and reducing the chance of the patient being sucked into the secondary health care system with all its emotional and financial consequences. This is a strategy along official governmental policy lines [36]. Yet, as appeared throughout the course of the study, the neurologists took a conservative approach and were inclined to return the patients quickly to the GP. Even though the randomization had been by the physicians, there might have been a carry-over effect through the attending neurologists as they were aware of the goal to reduce health care utilization in this group of patients. In fact, the period of the design of the study resulted in a first formal contact between the department of psychiatry and neurology and it touched a latent problem: how to approach patients where you cannot find a physical explanation for their complaint. Consequently, this might have been an additional factor reducing the estimated effect size.

A third factor to be considered is the specificity of the intervention. In this study the focus was the enhancement of the communication between the providers (neurologist and GP). In somatizing patients, who are inclined to have a more extensive health care utilization, an intervention focusing on the continuity of care can be regarded as specific, as lack of communication might result in redundancy of care. Yet for the patients with a high psychological distress score, a psychiatric co-treatment or a series of conjoint sessions with both the GP and the $\mathrm{C}-\mathrm{L}$ psychiatrist involved might have been more specific, a third generation intervention according to Katon's classification.

The target group of LBP patients in this sample was very diverse [37]. In a parallel study with the same intervention in the case of outpatients with abdominal pain (internal medicine), more positive patient and physician effects have been demonstrated [33,38]. At follow-up after 6 months, there was a significantly greater decline in depressive (SCL-90) and psychiatric (GHQ-28) symptoms in the abdominal pain patient intervention group compared with the control group. Also the GPs in the intervention group were significantly more satisfied with the cooperation and communication with the internists compared with the control group. The degree of implementation of the protocol contributed further to this. A rationale for this effect might be that in case of abdominal pain patients, psychiatric comorbidity is more clear compared with LBP patients [39]. Maybe the effectiveness of protocol-driven interventions for a specific co-morbid condition such as depression in case of LBP-patients, would have a stronger likelihood of effectiveness.

In conclusion, first the modest effects of the study should be seen in the perspective of type II errors, i.e., the level, intensity, and specificity of the intervention. Second, the intervention used is only one part of the intervention to be provided to this patient group: the enhancement of collaboration between secondary and primary care. In addition, a "third generation" intervention might be considered in the high-distressed group. Yet, probably due to the possibility of spontaneous remission, this might only be offered to patients not reassured by the assessment of the neurologist. Third, these intervention studies will have a better chance of reducing unnecessary medical care utilization when there is an indication for a redundancy of care. If the extent of the care provided is restricted, a reduction in the level of health care utilization is difficult to observe. Fourth, C-L psychiatrists should be aware of the fact that only modest and gradually increasing effects can be expected of intervention studies in such a complex area of health service research [20,34].

In this study, the medical specialists had a pivotal role, for example, in initiating the liaison with the GPs. In addition, we believe that the GP could also play a more central role in a holistic approach to the treatment of LBP patients, particularly in case of chronicity. Whether the liaison model described in this study can be used in routine clinical practice elsewhere, will need to be determined. If it is possible it is likely to result in GPs being more satisfied with their involvement with secondary services. Currently, the possibility of conjoint-consultations (C-L psychiatrist and GP) in these types of patients are being assessed [40].

This study was conducted as part of a larger ongoing project entitled "Interaction between primary and secondary health care" (Free University Hospital, Amsterdam, The Netherlands). In addition to the authors, participating members of the research group included $Y$. Dolstra, GP; A. J. M. Donker, internist; R. van Dyck, psychiatrist; F. J.M. Meiland, psychologist; J. G. Streefkerk, GP. We wish to thank the patients and physicians who took part in this study, and R. van Geel and 
H. Linssen, psychologists, for their assistance in performing the statistical analyses.

\section{References}

1. Foets $\mathrm{M}$, Sixma $\mathrm{H}$ : Een nationale studie van ziekte en verrichtingen in de huisartspraktijk. Basisrapport: Gezondheid en gezondheidsgedrag in de praktijkpopulatie (A national study of morbidity patterns in general practice. Basic report: health and health behavior). Utrecht, NIVEL, 1991

2. Linssen C: Pijnbeheersing: Een cursus voor mensen met rugpijn (Coping with pain: a course for people with back pain) (Dissertation). Leiden, State University Leiden, 1990

3. Frymoyer JW: Bain pain and sciatica. $N$ Engl J Med 318:291-300, 1988

4. Waddell G: Biopsychosocial analysis of low back pain. Baillieres Clin Rheumatol 6:523-557, 1992

5. Atkinson JH, Slater MA, Patterson TL, Grant I, Garfin SR: Prevalence, onset, and risk of psychiatric disorders in men with chronic low back pain: a controlled study. Pain 45:111-121, 1991

6. Manning WG, Wells KB: The effects of psychological distress and psychological well-being on use of medical services. Med Care 30:541-553, 1992

7. Levenson JL, Hamer RM, Rossiter LF: Relation of psychopathology in general medical patients to use and cost of services. Am J Psychiatry 147:1498-1503, 1990

8. Saravay SM, Strain JJ: Reviews of outcome studies: introduction. Psychosomatics 35:227-232, 1994

9. Love AW, Peck CL: The MMPI and psychological factors in chronic low back pain: a review. Pain 28:1-12, 1987

10. Keel PJ: Psychosocial criteria for patient selection: review of studies and concepts for understanding chronic back pain. Neurosurgery 15:935-941, 1984

11. Mumford E, Schlesinger H], Glass GV, Patrick C, Cuerdon T: A new look at evidence about reduced cost of medical utilisation following mental health treatment. Am J Psychiatry 141:1145-1158, 1984

12. Smith GR Jr, Monson RA, Derby CR: Psychiatric consultation in somatization disorder: a randomized controlled study. N Engl J Med 314:1407-1413, 1986

13. Katon $W$, Von Korff $M$, Lin E, et al: A randomized trial of psychiatric consultation with distressed highutilizers. Gen Hosp Psychiatry 14:86-98, 1992

14. Kamerov D, Burns B: The effectiveness of mental health consultation and referral in ambulatory primary care: a research lacuna. Gen Hosp Psychiatry 9:111-117, 1987

15. Huyse FJ, Hengeveld MW: The development of $\mathrm{C} / \mathrm{L}$ psychiatry in the Netherlands: its social psychiatric heritage. Gen Hosp Psychiatry 11:9-15, 1989

16. Bakers J, Burg-van Walsum $M$ van der: Medische contactdag Breda: Huisartsen en specialisten over hun onderlinge communicatie (Medical contact day Breda: GPs and specialists on their mutual communication). Medisch Contact 43:372-374, 1988

17. Bridges KW, Goldberg DP: Somatic presentation of
DSM-III psychiatric disorders in primary care. I Psychosom Res 29:563-569, 1985

18. Pincus HA: Patient oriented models for linking primary care and mental health care. Gen Hosp Psychiatry 9:95-101, 1987

19. Strathdee G: Primary care-psychiatry interaction: a British perspective. Gen Hosp Psychiatry 9:102-110. 1987

20. Katon W, Gonzales J: A review of randomized trials of psychiatric consultation liaison studies in primary care. Psychosomatics 35:268-278, 1994

21. Smith GR: The course of somatization and its effects on health care utilization. Psychosomatics 35:263267,1994

22. Engel GL: The need for a new medical model: a challenge for biomedicine. Science 196:129-136, 1977

23. American Psychiatric Association: Diagnostic and Statistical Manual of Mental Disorders, 3rd ed., revised. Washington, American Psychiatric Association, 1987

24. Aaronson NK, Bullinger M, Ahmedzai s: A modular approach to quality-of-life assessment in cancer clinical trials. Recent results in cancer research 111:231249,1988

25. Goldberg D, Williams P: A user's guide to the General Health Questionnaire. Windsor, Berkshire, U.K., NFER-NELSON, 1988

26. Koeter MWJ, Ormel J: De Nederlandse handleiding voor de General Health Questionnaire (The Dutch Manual of the General Health Questionnaire). Lisse, Swets \& Zeitlinger, 1991

27. Arrindell WA, Ettema JHM: SCL-90: Handleiding bij een multidimensionele psychopatholugie-indicator (SCL-90: manual of a multi-dimensional psychopathology indicator). Lisse, Swets Test Services, 1986

28. Huyse FJ, Strain JJ, Hengeveld MW: Interventions in consultation-liaison psychiatry: the development of a scheme and a checklist for operationalized interventions. Gen Hosp Psychiatry 10:88-101, 1988

29. Huyse FJ, Strain JJ, Hammer JS: Interventions in consultation/liaison $(C / L)$ psychiatry, part II: concordance. Gen Hosp Psychiatry 12:221-231, 1990

30. Huyse FI, Lyons JS, Strain JJ: Evaluating psychiatric consultations in the general hospital: multivariate prediction of concordance. Gen Hosp Psychiatry 14: 363-369, 1992

31. Stevens L: Applied multivariate statistics for the social sciences. Hillsdale, New Jersev, Lawrence Erlbaum, 1992

32. Lubo A, Pérez-Echeverría $M-J$, Jiménez-Aznárez $A$, Sancho M-A: Emotional disturbances in endocrine patients: validity of the scaled version of the GHQ-28. Br J Psychiatry 152:807-812, 1988

33. Meeuwesen L, Huyse FJ, Meiland FJM, Koopmans GT, Donker AJM: Psychiatric consultations in medical outpatients with abdominal pain: patient and physician effects. Int J Psychiatry Med 24:339-356, 1994

34. Starfield B: Health service research: a working model. $\mathrm{N}$ Engl J Med 289:132-136, 1973

35. Lyons JS, Hammer JS, Larson DB, Petraitis J, Strain JJ: Treatment opportunities on a C-L service. Am J Psychiatry $145: 1435-1437,1988$ 
36. Borst-Filers E: Nota modernisering curatieve zorg (Memorandum modernization curative care). 's-Gravenhage, Ministerie van Volksgezondheid, Welzijn en Sport, 1995

37. Koopmans GT, Meeuwesen L, Huyse FJ, Heimans JJ: Effects of psychiatric consultation on medical consumption in medical outpatients with low-back pain. Gen Hosp Psychiatry 18:145-154, 1996

38. Koopmans GT, Meeuwesen L, Huyse FJ, Meiland FJM, Donker AJM: Effects of psychiatric consultation on medical consumption in medical outpatients with abdominal pain. Psychosomatics 36:387-399, 1995

39. Creed F, Guthrie E: Psychological treatments of the irritable bowel syndrome: a review. Gut 30:16011609,1989

40. van der Feltz-Cornelis CM, Wijkel D, Verhaak PFM, Collijn DH, Huyse FJ, van Dyck R: Psychiatric consultation in somatizing patients in the family practice setting: a feasibility study. Int J Psychiatry Med 26 (in press) 